\title{
The Importance of Dorsoulnar Flap in The Thumb's Pulp Reconstruction - A Case Report and a Review of the Literature
}

\author{
Rekik MA*, Abid A, Ayadi W, Mohamed BJ, Bouaziz W, Zribi W, Ellouz Z, Ayadi K and Keskes H \\ Orthopedics Department, Habib Bourguiba University Hospital, Sfax, Tunisia
}

*Corresponding author: Rekik MA, Orthopedics Department, Habib Bourguiba University Hospital, Sfax, Tunisia, Tel: 0021622838381, E-mail: rekikmohamedali86@gmail.com

Citation: Rekik MA, Abid A, Ayadi W, Mohamed BJ, Bouaziz W, et al. (2018) The Importance of Dorsoulnar Flap in The Thumb's Pulp Reconstruction - A Case Report and a Review of the Literature. J Surg Oper Care 3(2): 204. doi: 10.15744/2455-7617.3.204

Received Date: March 26, 2018 Accepted Date: July 17,2018 Published Date: July 20,2018

\begin{abstract}
The arterial supply of the thumb differs greatly from that of the other fingers. The surgical technique is based on the presence of the ulnar dorsal digital artery of the thumb. By reference to anatomical facts, we present a case of simple local flap, a dorsoulnar flap of the thumb, which presents a possibility to cover the extensive distal and pulpal thumb's defects. As results, the flap was viable, well vascularized and the coverage was good.

Keywords: Flap; Thumb; Reconstruction.
\end{abstract}

\section{Introduction}

The arterial supply of the thumb differs greatly from that of the other fingers. The thumb's dorsal collateral arteries are the subject of comprehensive studies. The extensive loss of the thumb's pulp with or without bone exposure is a major problem for the patient and remains a challenge to take up for the surgeon often brought to cover the losses using local and remote flaps. The ulnar dorsal digital artery of the thumb, which originates from the palmar arteries at the head of the first metacarpal bone, continues distally via the proximal nail fold arcade, and is nourished by the palmar anastomosis at the level of the neck of the proximal phalanx. By reference to anatomical facts (1), we present a case of simple local flap, a dorsoulnar flap of the thumb, which presents a possibility to cover the extensive distal and pulpal thumb's defects.

\section{Purpose}

We present a case of a reconstruction of an extensive thumb's pulpal defect using the dorsoulnar flap specifyin its technique and its therapeutic interest.

\section{Material and Methods}

We report a case of a 54 years old, right handed, female patient, working as a baker presenting with a decay of the palmar face of the distal phalanx of the right thumb related to a work accident (Figure 1). The lesion exposed the bone, which motivated the use of a pedicle flap to cover the defect

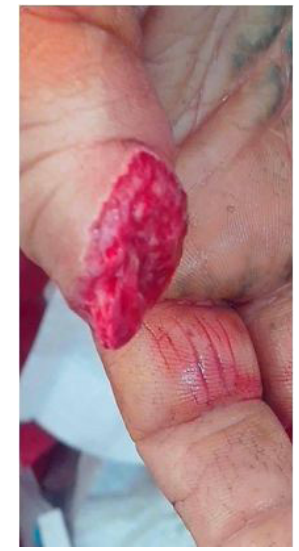

Figure 1: Preoperative picture of the thumb 


\section{Operative Technique}

The skin is first marked as follows (Figure 2) the dorsal arcade of the proximal nail fold (at $0.9 \mathrm{~cm}$ proximal to the cuticle), the palmar anastomosis at the level of the neck of the first phalanx $(2.5 \mathrm{~cm}$ proximal to the cuticle), and the dorsoulnar collateral artery at the level of the neck of the first phalanx from the median axis of the thumb) [1]. The subcutaneous flap pedicle area (that will be raised with the flap) is also marked on the skin. The flap is raised in a proximal to distal direction along the dorsoulnar arterial axis to avoid damaging the pedicle (Figure 3). During dissection, the subcutaneous tissue is harvested en bloc around the axis of the flap at a width of $1 \mathrm{~cm}$, leaving the aponeurosis in situ and without dissecting for the artery, which can be extremely small and therefore easily damaged. Dissection of the flap is continued to the level at middle of the proximal phalanx to preserve the anastomosis between palmar and dorsal vessels (Figure 4). The ulnar dorsal digital nerve was coapted to one of the volar digital nerves. The flap is then rotated and the skin paddle is sutured with a few absorbable stitches (Figure 5). The donor site is either primarily closed.

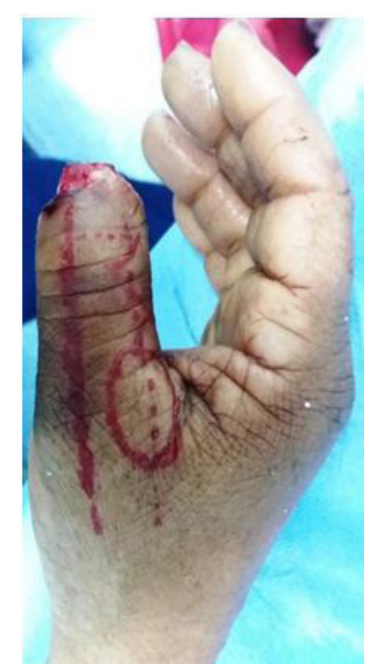

Figure 2: Operative incision

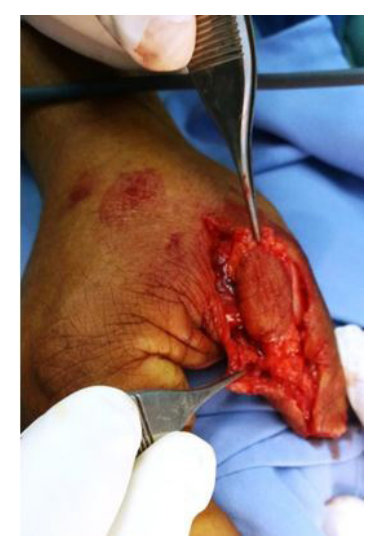

Figure 3: Peroperative view demonstrating the exposure of the subcutaneous pedicle

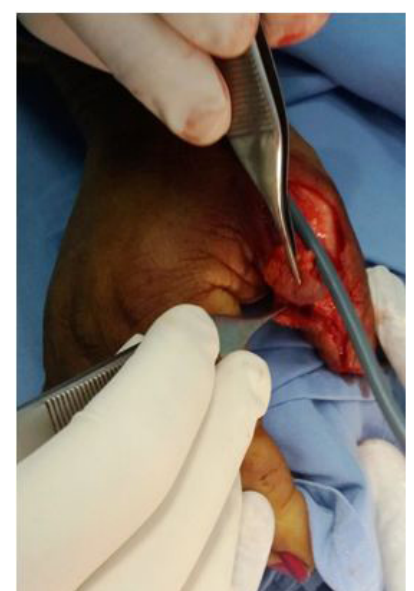

Figure 4: Peroperative view demonstrating the flap 


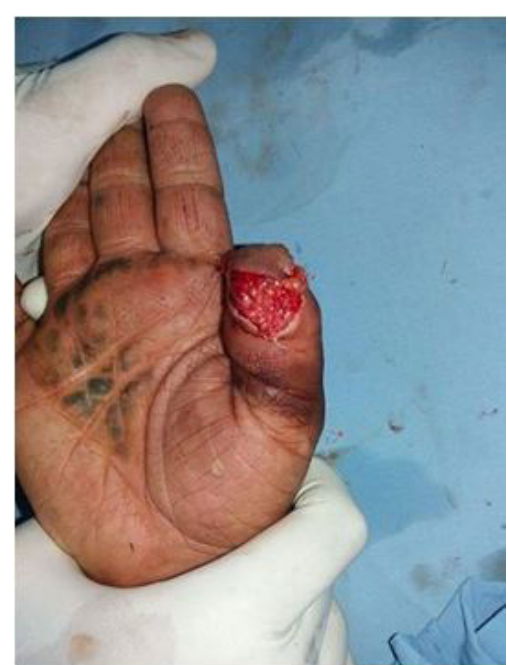

Figure 5: Postoperative view

\section{Results}

The flap was viable, well vascularized and the coverage was good (Figure 6). The operating follow ups were simple and no major complications happened (infection, necrosis, and neuroma) yet the flap's sensitivity was mediocre (Figure 7,8). The patient was last seen after 6 months and was satisfied with the final result with a good esthetical and functional result (Figure 9, 10, 11). A protective sensitivity was noticed.

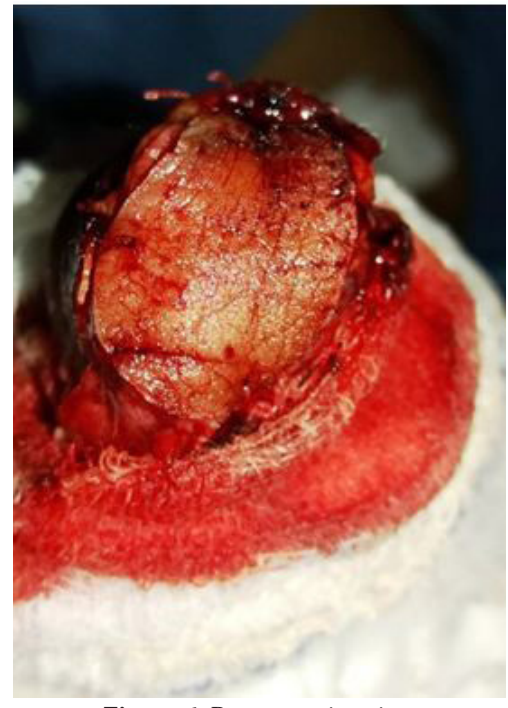

Figure 6: Postoperative view

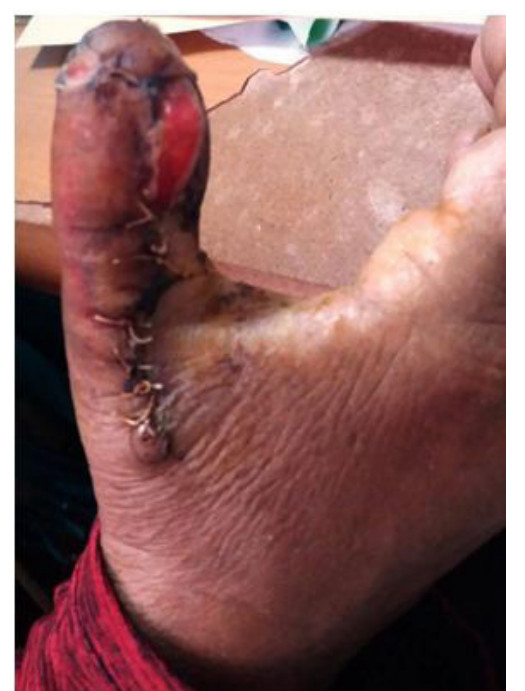

Figure 7: Clinical appearance at 15 days 


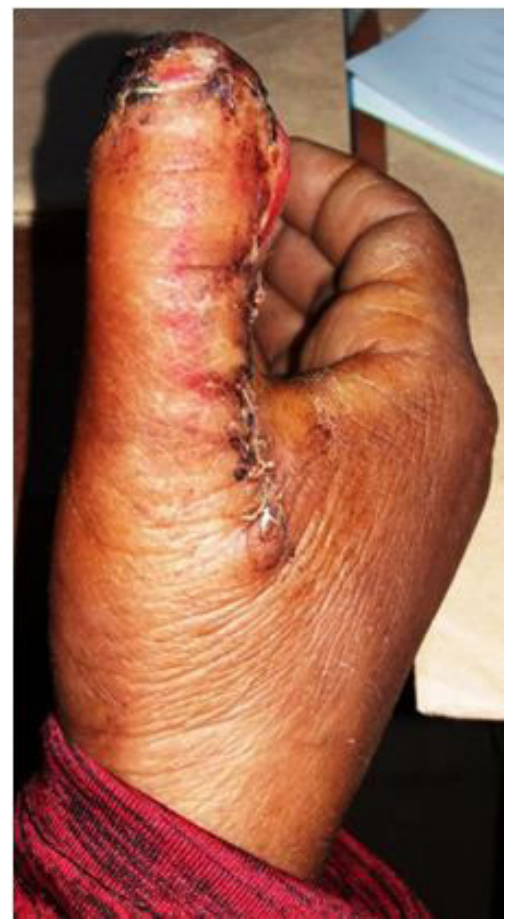

Figure 8: Clinical appearance at 15 days

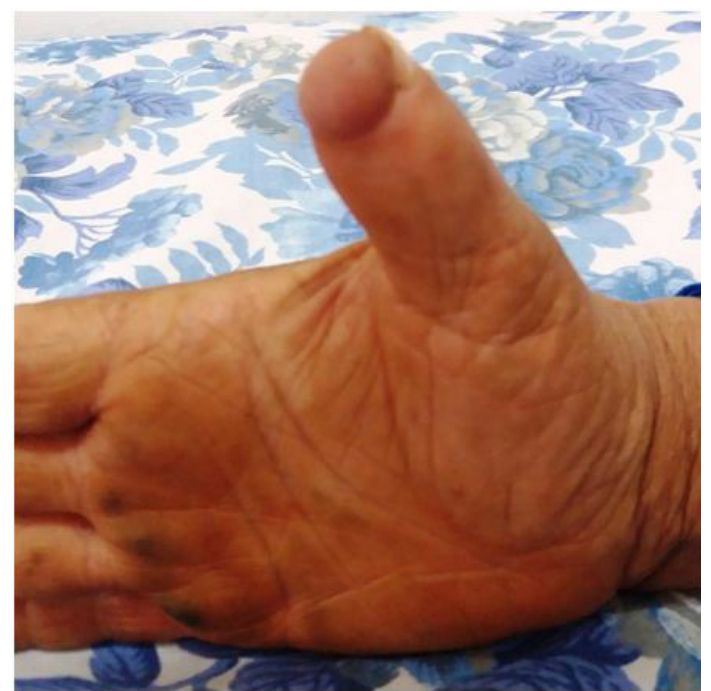

Figure 9: Clinical appearance of the thumb at 06 months

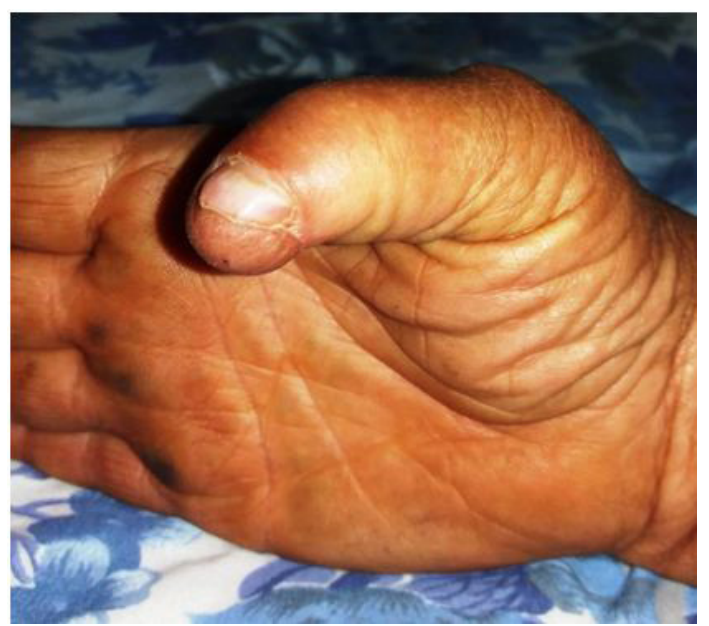

Figure 10: Clinical appearance and function at 06 months 


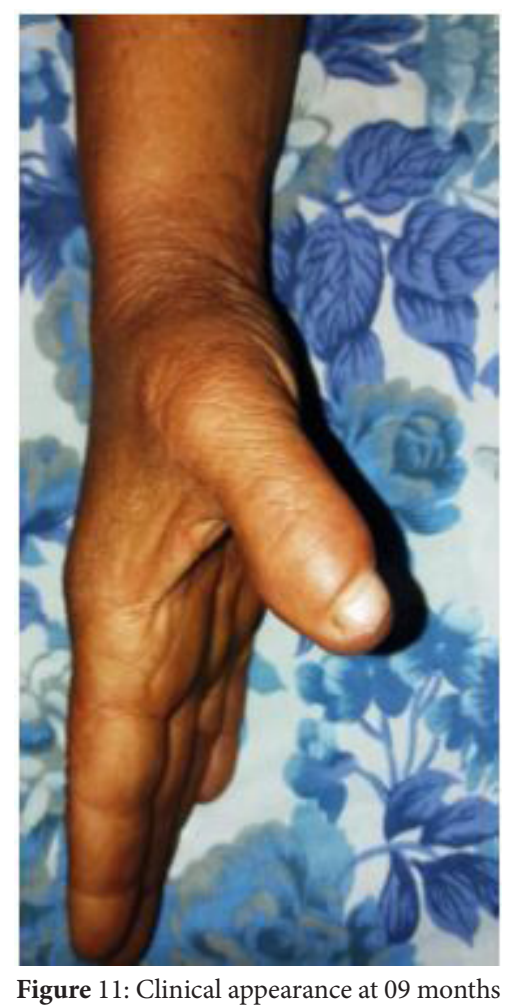

\section{Discussion}

In the thumb the dorsal arteries are quite well developed (especially on the ulnar side) and represented by an independent vascular axis that runs along the thumb; in the fingers they are inconstant, fragmentary, and dependent on the palmar collateral arteries [2]. Brunelli Published an anatomic study of the dorsoulnar flap of the thumb in 1991 and the first clinical application was carried out by the same author in 1993, a versatile fascio-cutaneous flap for fingertip soft tissue reconstruction of the thumb [3,4]. The Brunelli flap is a suitable technique in the treatment of injuries with small and moderate soft tissue defects of the volar side of the thumb. It is a relatively simple surgical procedure that leaves no major scarring. The drawback of this procedure is the lack of sensory recovery. The problem of sensibility of the thumb pulp has not yet been resolved, however, neither by heterodactyl island flaps nor by free sensory flaps [5].

\section{Conclusion}

The dorsoulnar flap of the thumb is a distally based island flap. Thanks to the negligible esthetical and functional sequelae in the donor region, because of the distal nature of its pedicle, this flap can be used in the distal reconstructions of the thumb's extensive distal soft tissue's loss.

\section{References}

1. Khan MM, Yaseen M, Bariar LM, Khan SM (2009) Clinical study of dorsal ulnar artery flap in hand reconstruction. Indian J Plast Surg $42: 52-7$.

2. Ameziane L, Manouar E Le Lambeau Dorso-Cubital Du Pouce.

3. Brunelli F, Pegin Z, Cabral J (1991) Dorsal arterial supply to the thumb. New surgical possibilities for palmar skin coverage. Surg Radiol Anat 13: 240-2.

4. Brunelli F (1993) Le lambeau dorso-cubital du pouce. Ann Chir Main Memb Supér 12: 105-4.

5. Coninck de A (1975) Heterodigital transplantation with local reinnervation. Acta Orthop Belg 41: 170-6. 


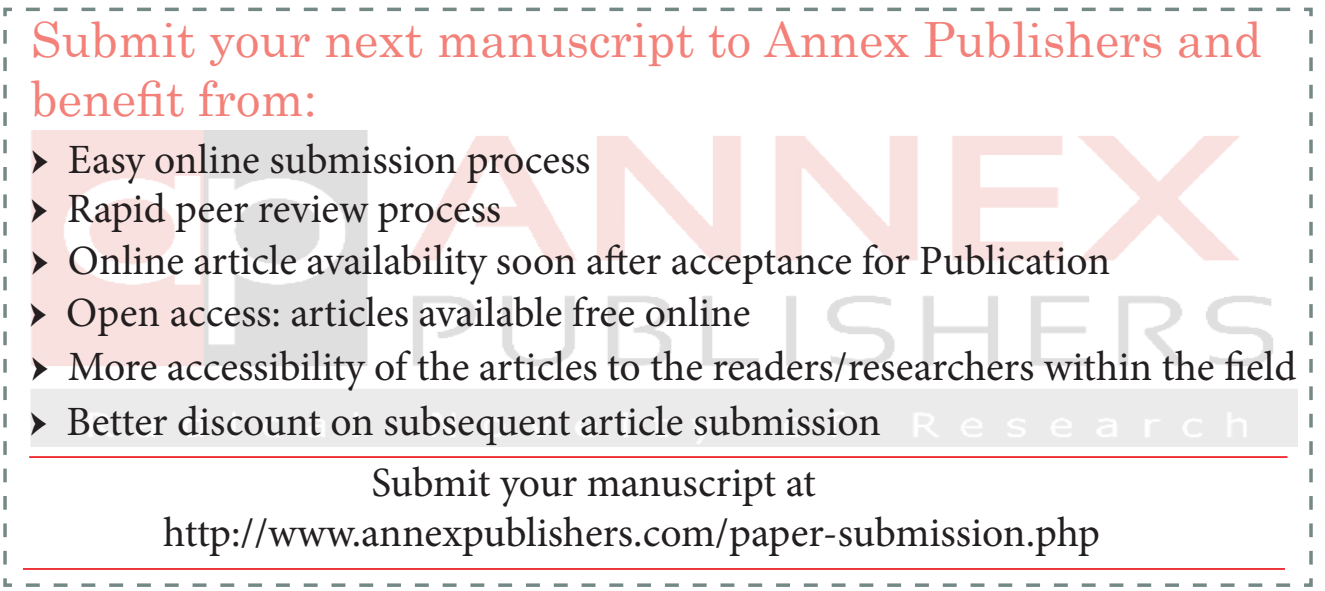

